\title{
Strategy for Reflector Pattern Calculation: Let the Computer Do the Work
}

P.T. Lam and S.W. Lee

University of Illinois

Urbana, Illinois

C.C. Hung

Lockheed Missiles and Space Company

Sunnyvale, California

and

R. Acosta

Lewis Research Center

Cleveland, Ohio

October 1985

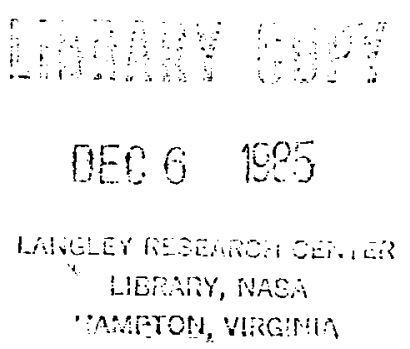




\title{
STRATEGY FOR REFLECTOR PATTERN CALCULATION:
}

\section{LET THE COMPUTER DO THE WORK}

\author{
by P.T. Lam and S.W. Lee \\ Electromagnetics Laboratory \\ Department of Electrical and Computer Engineering \\ University of illinois \\ Urbana, Illinois \\ C.C. Hung \\ Lockheed Missiles and Space Company \\ Sunnyvale, California \\ and R. Acosta \\ National Aeronautics and Space Administration \\ Lewis Research Center \\ Cleveland, Ohto 44135
}

\section{SUMMARY}

Using high-frequency approximations, the secondary pattern of a reflector antenna can be calculated by numerically evaluating a radiation integral $I(u, v)$. In recent years, tremendous effort has been expended to reducing $I(u, v)$ to Fourier integrals. These reduction schemes are invariably reflector geometry dependent. Hence, different analyses/computer software development must be carried out for different reflector shapes/boundaries. The purpose of this note is to point out, that, as the computer power improves, these reduction schemes are no longer necessary. Comparable accuracy and computation time can be achieved by evaluating $I(u, v)$ by a brute-force FFT described in this note. Furthermore, there is virtually no restriction on the reflector geometry by using the brute-force FFT.

\section{RADIATION INTEGRAL}

In calculating the radiation pattern of a reflector antenna, the central step is to evaluate the following radiation integral:

$$
I(u, v)=\iint_{\Sigma} J(x, y) e^{j k(u x+v y+w z)} d x d y
$$

where,

$$
\begin{aligned}
& (\theta, \phi) \text { observation direction } \\
& k=2 \pi / \lambda=\text { wave number } \\
& u=\sin \theta \cos \phi \\
& v=\sin \theta \sin \phi \\
& w=\cos \theta=\sqrt{1-\left(u^{2}+v^{2}\right)}
\end{aligned}
$$


$z=f(x, y)$ (equation describing the refiector surface)

$\Sigma=$ projection of the reflector on the $x y$ plane (fig. $1(b)$ ).

We note that equation (1) is not a two-dimensional Fourier integral because of the presence of the factor $\exp (j k w z)$, which reads explicitiy

$$
\exp \left(j k \sqrt{1-\left(u^{2}+v^{2}\right)} f(x, y)\right)
$$

In the past years, there has been a tremendous amount of effort to convert the radiation integral (1) to a Fourier integral. A popular method is the so-called "p-series expansion," in which the integral in equation (1) is expressed as

$$
I(u, v)=\sum_{p=0}^{\infty}\left(\cos \theta-\cos \theta_{0}\right)^{p} I_{p}(u, v)
$$

Here $\theta_{0}$ is the polar angle of the main beam (fig. 1(a)). Each integral $\mathrm{I}_{p}(u, v)$ in equation (3) is indeed a Fourier integral. Several clever schemes can be used for its evaluation (refs. 1 to 6 ). As an alternative to the p-series expansion, we may first calculate the aperture field over a planar surface by GO or GTD (refs. 7 and 8 ). Over the planar surface, $z$ is equal to a constant and the integral in equation (1) is reduced to a Fourier integral. Needless to say, either the p-series expansion or GO/GTD involves sophisticated mathematical manipulations.

While the above efforts are gratifying, they all suffer a drawback, namely, the analyses are heavily geometry dependent. Usually a given analysis falls if the reflector surfaces is changed from, for example, parabolic to hyperbolic, or the reflector boundary from circular to trapezoidal. As a consequence, one needs to develop different versions of computer code for different reflector geometries.

\section{BRUTE-FORCE FFT}

The purpose of this note is to point out that, because of the rapid progress of computer power, it is no longer advantageous to reduce equation (1) to a Fourier integral before going to a computer. We evaluate equation (1) by a brute-force FFT in the following manner:

(a) Represent $I(U, v)$ in terms of a sinc series, namely,

$$
I(u, v)=\sum_{m=-M+1}^{M} \sum_{n=-N+1}^{N} I_{m n} \frac{\sin (\pi(u A-m)}{\pi(u A-m)} \cdot \frac{\sin (\pi(v B-n))}{\pi(v B-n)}
$$


where

$$
\begin{aligned}
I_{m n} & =\frac{A B}{4 M N} e^{j 2 \pi\left(u x_{1}+v y_{1}\right)} F_{m n} e^{j 2 \pi(u A-m+v B-n)} \\
F_{m n} & =\text { Fourier series coefficients of } J(x, y) e^{k j W z}
\end{aligned}
$$

Note that the coefficients $\left\{I_{m n}\right\}$ are values of $I(u, v)$ at sampling points

$$
u=u_{m}=m / A, \quad v=v_{n}=n / B
$$

(b) Use the FFT to find $\left\{I_{m n}\right\}$ from equation (1). Since equation (1) is not a Fourier integral, we must apply the FFT once for each and every $I_{m n}$. Thus, we apply the FFT $2 M$ by $2 \mathrm{~N}$ times instead of once, if equation (1) was a Fourier integral. Fortunately, modern computers can perform the FFT operation very efficientiy.

\section{ACCURACY AND COMPUTATION TIME}

To 1 llustrate the accuracy and efficiency of the brute-force FFT method, the secondary pattern of an offset parabolic reflector with a circular aperture (fig. 2) was computed using the brute-force FFT, GO (ref. 7), GTD (ref. 7), and Fourier-Besse1 (ref. 1) methods. In each method, a 48 by 48 FFT grid ( $f$ ig. $1(b)$ ) was taken and the secondary pattern was calculated at 101 observation points. As expected, the secondary patterns calculated by each method are in good agreement. Taking a closer look, the gain and peak sidelobe levels for the first six sidelobes are tabulated for each method in table I. These values are in good agreement with the results calculated using the JacobiBessel series method (ref. 5). Another example with an off-focus feed is shown in figure 3, where good agreements among al1 methods are again observed.

Table II shows the computation times of the methods on a CYBER 175 computer. We note that the computation time of the brute-force FFT is of the same order of magnitude as the computation times using GO, GTD, and the Fourier-Bessel series method. For nonparabolic reflectors, the coefficients of the p-series method must be computed for every observation point. Hence, the computation times using the p-series are of the same order as the bruteforce FFT. The computation times using GO and GTD may also increase due to the possibility of more specular points present for a nonparabolic reflector.

\section{CONCLUSION}

1. Unlike other methods described in the literature, the brute-force FFT is most general in the sense that it applies to reflector with arbitrary shape and boundary. It is an ideal method for developing a user-friendly general purpose reflector computer program.

2. Within the high-frequency approximation used in all reflector analyses, the brute-force FFT is just as accurate as the other methods. 
3. Its computation time is in the same order of magnitude as those of other methods. In the worst case (a parabolic reflector with circular aperture), it is three times slower than other methods that take advantage of the special geometry and use clever mathematical manipulations.

4. The above time comparison is based on the fact that the FFT is performed using a software subroutine. For the cyber 175 computer, the FFT computed using an array processor is five times faster than that using a software subroutine. Our message is that, as computer power improves, the brute-force FFT for reflector analysis will become more and more attractive.

\section{ACKNOWLEDGEMENT}

We would like to thank Dr. Yayha Rahmat-Samil of JPL for many valuable comments and for computing some of the data shown in table $I$.

\section{REFERENCES}

1. Hung, C.C.; and Mittra, R.: Secondary pattern and focal region distribution of reflector antennas under wide-angle scanning. IEEE Trans. Antennas Propag., vol. 31, no. 5, Sept. 1983, pp. 756-763.

2. Galindo-Israel, V.; and Mittra, R.: A new series representation for the radiation integral with application to reflector antennas. IEEE Trans. Antennas Propag., vol. 25, no. 5, Sept. 1977, pp. 631-641.

3. Mittra, R., et al.: An efficient technique for the computation of vector secondary patterns of offset paraboloid reflectors. IEEE Trans. Antennas Propag., vol. 27, no. 3, May 1979, pp. 294-304.

4. Hung, C.C.: Fourier-Bessel series representation for the far field pattern of arbitrary reflector antennas. LMSC Report 844955, Lockheed Missiles and Space Company, Sunnyvale, CA, 1982.

5. Rahmat-Sami\}, Y.; Galindo-Israel, V.: Shaped reflector antenna analysis using the Jacobi-Bessel series. IEEE Trans. Antennas Propag., vol. 28, no. 4, July 1980, pp. 425-435.

6. Hung, C.C.; and Mittra, R.: Fourier-Bessel technique for analyzing spherical reflector antennas. Third International Conference on Antennas and Propagation, (ICAP-83), Pt. 1, IEE CP-219, Institue of Electrical Engineers, Stevenage, England, 1983, pp. 433-435.

7. Lam, P.T.; Lee, S.W.; and Acosta, R.: Secondary pattern computation of an arbitrarily shaped main reflector. Electromagnetics Laboratory, University of Illinois, Urbana, IL, 1984.

8. Chang, Y.C.; and Rudduck, R.C.: Extended aperture integration for the analysis of offset reflector antennas. 1984 International Symposium Digest on Antennas and Propagation, vol. 1, IEEE, 1984, pp. 293-296. 
TABLE I. - GAIN AND SIDELOBE LEVELS OF OFFSET PARABOLIC REFLECTOR COMPUTED USING SEVERAL DIFFERENT METHODS

\begin{tabular}{|l|c|c|c|c|c|}
\hline & $\begin{array}{c}\text { Jacobi-Besse1, } \\
\text { ref.5 }\end{array}$ & FFT & $\begin{array}{c}\text { GTD } \\
\text { ref.7 }\end{array}$ & $\begin{array}{c}\text { GO } \\
\text { ref. 7 }\end{array}$ & $\begin{array}{c}\text { ourter-Besse1, } \\
\text { ref. 7 }\end{array}$ \\
\hline Gain (dB) & 48.28 & 48.25 & 48.31 & 48.29 & 48.22 \\
1st SL & 28.70 & 28.94 & 28.48 & 28.49 & 27.98 \\
2nd SL & 22.50 & 22.48 & 22.64 & 22.78 & 21.92 \\
3rd SL & 18.32 & 18.85 & 17.72 & 17.63 & 17.76 \\
4th SL & 15.21 & 15.55 & 15.80 & 14.93 & 14.64 \\
5th SL & 12.67 & 12.76 & 12.09 & 8.54 & 12.14 \\
6th SL & 10.55 & 11.15 & 11.30 & 9.96 & 10.01 \\
\hline
\end{tabular}

TABLE II. - EXECUTIVE TIME OF SEVERAL

DIFFERENT METHODS ON A CYBER 175 COMPUTER TO COMPUTE SECONDARY PATTERN

\begin{tabular}{|c|c|c|c|c|}
\hline \multicolumn{1}{|c|}{ Method } & FFT & GTO & GO & Fourier-Besse $)$ \\
\hline $\begin{array}{l}\text { executive time } \\
\text { on CYBER (CPU sec) }\end{array}$ & 62 & 73 & 22 & 22 \\
\hline
\end{tabular}



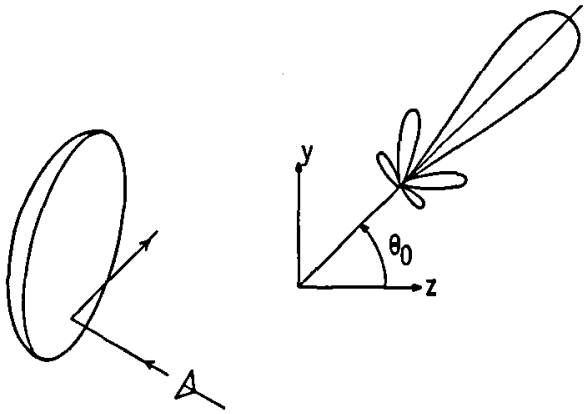

(a) Main beam reference (polar angle).

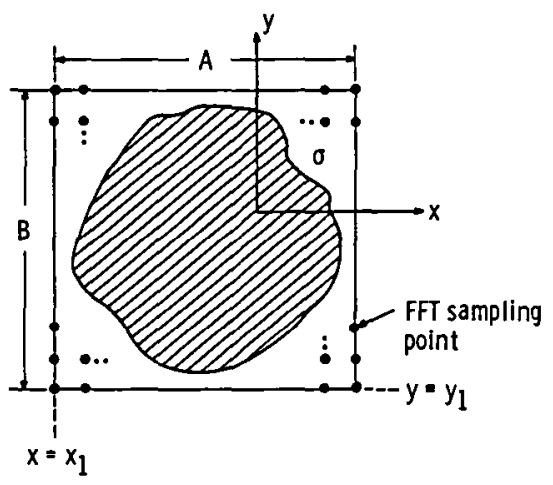

(b) Fourier aperture grid.

Figure 1. 


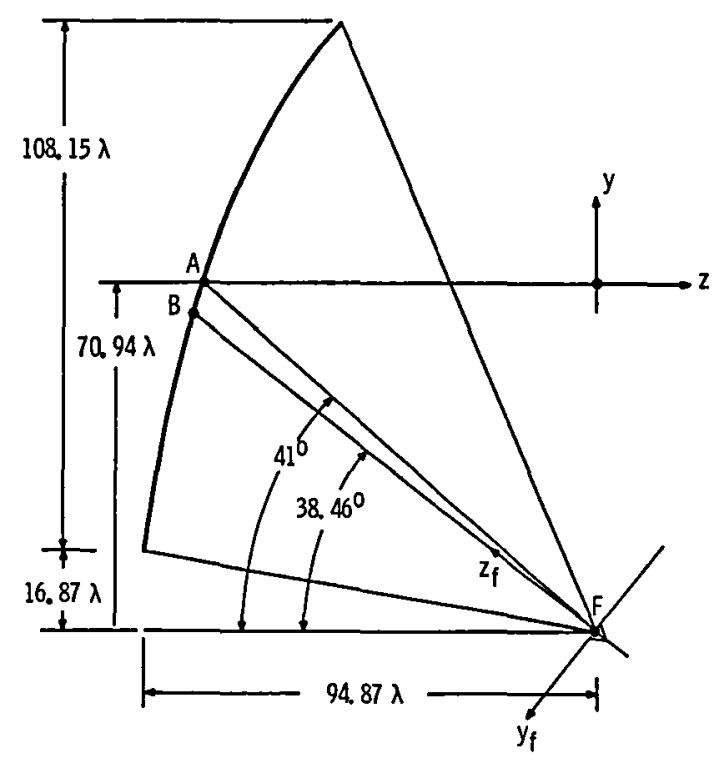

Figure 2. Offset parabolic reflector configuration.

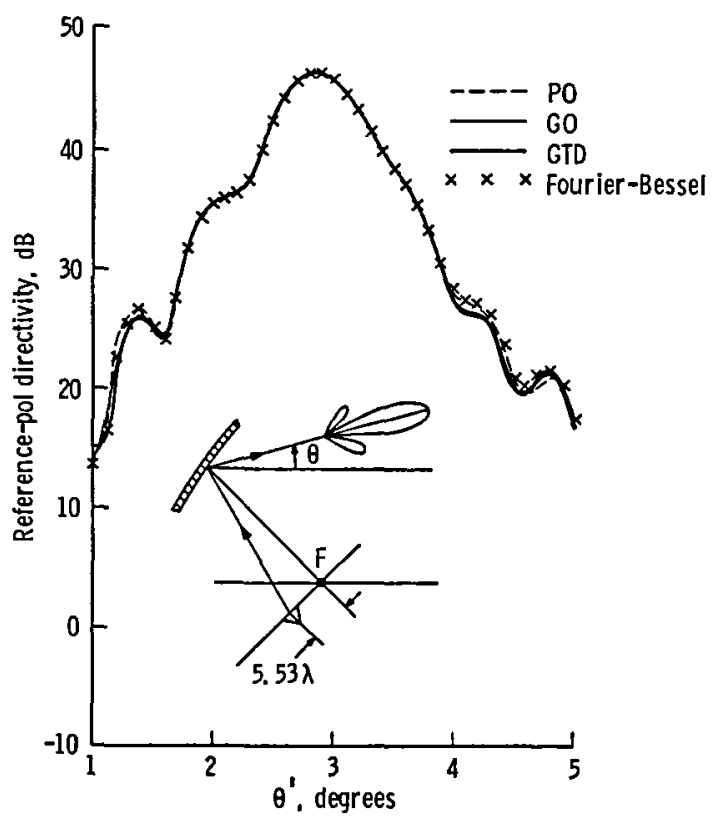

Figure 3. - Far-field antenna pattern comparison. 


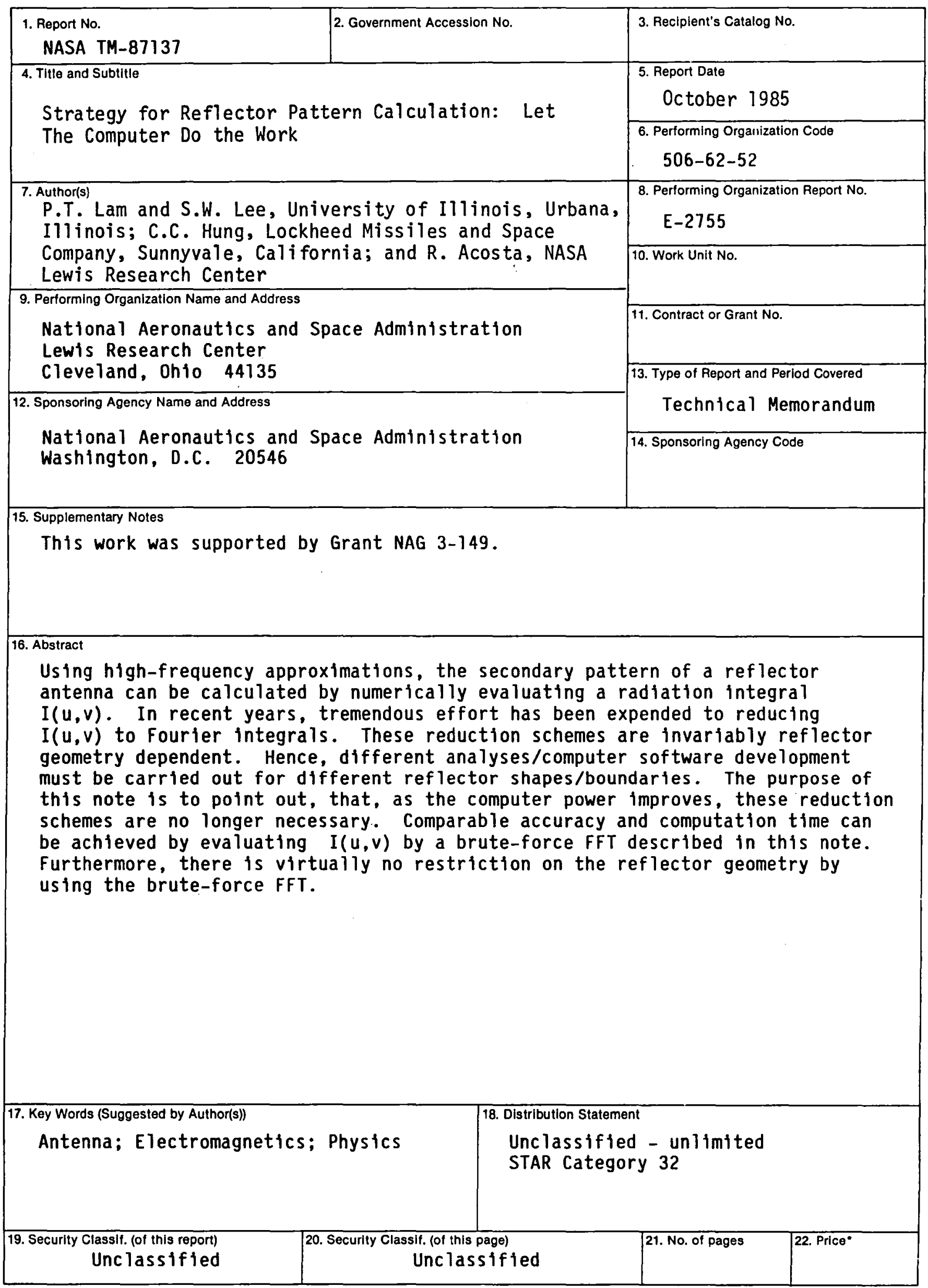

"For sale by the National Technical Information Service, Springfield, Virginia 22161 
End of Document 